\title{
Adetayo S. Eegunjobi* and Oluwole D. Makinde Second law analysis for MHD permeable channel flow with variable electrical conductivity and asymmetric Navier slips
}

\begin{abstract}
The inherent irreversibility in a steady hydromagnetic permeable channel flow of a conducting fluid with variable electrical conductivity and asymmetric Navier slip at the channel walls in the presence of induced electric field is theoretically investigated. The model nonlinear governing equations are obtained and numerically solved using shooting quadrature. Numerical results for velocity and temperature profiles are utilised to compute the entropy generation number and the Bejan number. Pertinent results are displayed graphically and discussed quantitatively.
\end{abstract}

Keywords: MHD channel flow; entropy generation; suction/injection; variable electrical conductivity; current density; asymmetric Navier slips

PACS: 47; $47.11 \mathrm{Hj}$

DOI 10.1515/phys-2015-0014

Received April 29, 2014; accepted September 26, 2014

\section{Nomenclature}

$c_{r} \quad$ Skin-Friction coefficient.

$P \quad$ Fluid pressure.

$u \quad$ Fluid velocity.

La Electrical field loading parameter.

$T \quad$ Fluid Temperature.

$E_{z} \quad$ Induced magnetic field.

Be Bejan number.

$(x, y) \quad$ Cartesian coordinates.

$T_{w} \quad$ Upper wall temperature.

$J_{z} \quad$ Current density.

a Channel width.

M Magnetic field parameter.

^Corresponding Author: Adetayo S. Eegunjobi: Department of Mathematics and Statistics, Namibia University of Science and Technology, Private Bag 13388, 13 Storch Street, Windhoek, Namibia, E-mail: samdet1@yahoo.com

Oluwole D. Makinde: Faculty of Military Science, Stellenbosch University, Private Bag X2, Saldanha 7395, South Africa, E-mail: makinded@gmail.com
Re Suction/Injection Reynolds number.

$I_{T} \quad$ Total current.

Ns Dimensionless form of entropy generation.

Ec Eckert Number.

$k \quad$ Thermal conductivity.

$E_{G} \quad$ Entropy generation.

$T_{0} \quad$ Lower wall temperature.

$G \quad$ Pressure gradient.

Pr Prandtl Number.

$\mathrm{Br} \quad$ Brinkmann Number.

$w \quad$ Dimensionless velocity.

$\mathrm{Nu} \quad$ Nusselt NUmber.

$B_{0} \quad$ Magnetic field.

\section{Greek letters}

$\theta \quad$ Dimensionless temperature.

$\beta_{0}, \beta_{1} \quad$ Slip coefficients.

$\sigma_{w} \quad$ Fluid electrical conductivity.

$\Phi \quad$ Irreversibility Ratio.

$\lambda \quad$ Electrical conductivity variation index.

$\Omega \quad$ Temperature difference parameter.

$\eta \quad$ Dimensionless transverse coordinate

$\gamma_{0}, \gamma_{1} \quad$ Dimensionless Navier Slip Coefficient.

\section{Introduction}

The study of the (Magnetohydrodynamic) MHD flow of a conducting fluid through a permeable channel between two parallel plates has gained the attention of scientists and engineers in the recent time. This is attributed to vital applications in various branches of industries and engineering such as MHD generators, MHD pumps, accelerators, electrostatic precipitation, polymer technology, Petroleum industries, purification of crude oil, plasma studies, nuclear reactors, geothermal energy extraction, the boundary control in the field of aerodynamics and blood flow problems. Based on the application, various mathematical models have been formulated to study the behavior of the flow. [1] examined the motion of a two dimensional steady flow of a viscous, electrically conducting, incompressible fluid flowing between two infinite parallel porous plates under the influence of transverse mag- 
netic field and constant pressure gradient. [2] studied the combined effect of a transverse magnetic field and radiative heat transfer to the unsteady flow of a conducting optically thin fluid through a channel filled with saturated porous medium and non-uniform wall temperature. [3] investigated the hydromagnetic steady flow of a viscous conducting fluid in a channel with slip at permeable boundaries. Several experimental works on the effects of magnetic fields of the flow and heat transfer characteristics of electrically conducting fluids can be found in [4-6] etc. Entropy generation is closely related to thermodynamic irreversibility, which is encountered in all heat transfer processes. The analysis of thermodynamic irreversibility paves the way to identify the irreversibility associated with various components and to avoid loss of available energy. $[7,8]$ was the first to introduce the theoretical concept of entropy generation minimization based on the secondlaw analysis into heat transfer and thermal design problem. After the pioneering works of $[7,8]$, several other researchers have discussed entropy generation under various conditions. [9] studied the combined effects of convective heating and suction/injection on the entropy generation in a steady flow of an incompressible viscous fluid through a channel with permeable walls. [10] numerically investigated the entropy generation due to steady laminar forced convection fluid flow through parallel plates microchannel. [11] analytically examined the effects of radiation heat transfer on magnetohydrodynamic mixed convection through a vertical channel packed with fluid saturated porous substances. [12] investigated the effects of the thermodynamic second law on steady flow of an incompressible variable viscosity electrically conducting fluid in a channel with permeable walls and with convective surface boundary conditions. Second law analysis is a new emergence in the field of radiation heat transfer (see $[13,14])$.

Recently, some researchers have shown interest in the study of convective transport of slip flow due to its applications. [15] studied the boundary layer flow over a flat plate with slip flow and constant heat flux surface conditions. [16] studied the combined effect of buoyancy force and Navier slip on entropy generation in a vertical porous channel. [17] modeled flow in a laminar boundary layer by using slip boundary conditions. [18] considered the effect of Navier slip on entropy generation in a porous channel with suction/injection. [19] investigated the driven cavity flow using the Navier slip boundary conditions.

In the present study, our objective is to study the inherent irreversibility in steady hydromagnetic permeable channel flow of a conducting fluid with variable electrical conductivity and asymmetric Navier slip at the channel walls in the presence of induced electric field. The paper is organised as follows: we define the problem, give the governing equations and present the mathematical formulation in Section 2. In Section 3, we derive the entropy generation rate and Bejan number while in Section 4, we present and discuss the pertinent results graphically and quantitatively. Finally, in Section 5, we make concluding remarks.

\section{Mathematical model}

The steady hydromagnetic flow of a viscous conducting incompressible fluid in a channel with permeable walls is considered in the presence of an asymmetric Navier slip, an imposed transverse magnetic field of strength $B_{0}$ and an induced electric field $E_{z}$. The induced magnetic field is assumed to be small when compared to the applied magnetic field and is thus neglected. Both fluid injection and suction are assumed to take place at the lower and upper walls respectively (shown in Figure 1 below). The channel wall temperature is non-uniform with temperature $T_{0}$ at the lower wall and $T_{w}$ at the upper wall such that $T_{w}>T_{0}$.

The temperature dependent variable electrical conductivity is given as ([20])

$$
\bar{\sigma}=\sigma_{w}\left(\frac{T-T_{0}}{T_{w}-T_{0}}\right)^{\lambda},
$$

where $\sigma_{w}$ is the fluid electrical conductivity at the upper plate and $\lambda$ is the electrical conductivity variation index. Under the above assumptions, the dimensionless governing equations for the momentum and energy balance can be expressed as:

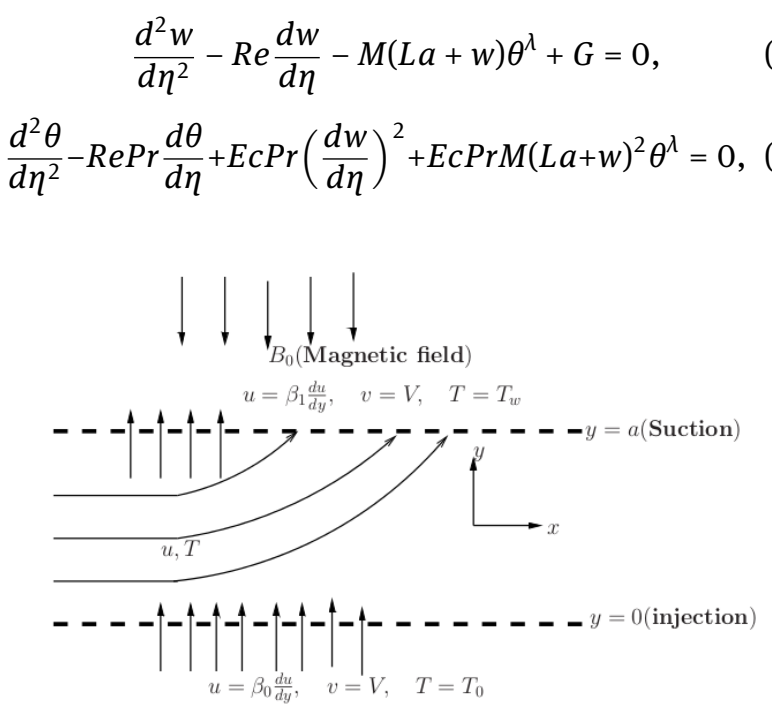

Figure 1: Geometry of the problem. 
where $\theta$ is the dimensionless temperature, w is the dimensionless velocity, $\beta_{0}$ and $\beta_{1}$ are slip coefficients, $R e, L a$, $M, E c, P r$, and $G$ are the suction/injection Reynolds number, electric field loading parameter, Magnetic field parameter, Eckert number, Prandtl number and the pressure gradient parameter. The appropriate boundary conditions in dimensionless form are given as follows:

$$
\begin{aligned}
& W(1)=\gamma_{1} \frac{d w}{d \eta}(1), \quad \theta(1)=1, \\
& W(0)=\gamma_{0} \frac{d w}{d \eta}(0), \quad \theta(0)=0 .
\end{aligned}
$$

The following quantities have been utilised in order to obtain the dimensionless governing equations (2)-(5):

$$
\begin{aligned}
& \eta=\frac{y}{a}, \quad X=\frac{x}{a}, \quad w=\frac{u}{V}, \quad L a=\frac{E_{z}}{V B_{0}}, \\
& \theta=\frac{\left(T-T_{0}\right)}{\left(T_{w}-T_{0}\right)}, \quad E c=\frac{V^{2}}{c_{p}\left(T_{w}-T_{0}\right)}, \\
& P=\frac{a \bar{P}}{\mu V}, \quad \operatorname{Pr}=\frac{\mu c_{p}}{k}, \quad \operatorname{Re}=\frac{\rho V a}{\mu}, \quad \gamma_{1}=\frac{\beta_{1}}{a}, \\
& \sigma=-\frac{\bar{\sigma}}{\sigma_{w}}, \quad G=-\frac{d P}{d X}, \quad M=\frac{\sigma_{w} B_{0}^{2} a^{2}}{\mu},
\end{aligned}
$$

where $T$ is the temperature, $V$ is the uniform suction / injection velocity, $a$ is the channel width, $k$ the thermal conductivity, $P$ is the fluid pressure, $\gamma_{0}$ and $\gamma_{1}$ is the Navier slip parameters for both the lower and upper walls respectively. The set of equations (2)-(3) under the boundary conditions (4) and (5) were numerically solved by applying the shooting technique together with a fourth-order Runge-Kutta-Fehlberg integration scheme ([21]). Both velocity and temperature profiles were numerically obtained and utilized to compute the skin-friction coefficient $\left(C_{f}\right)$ and the Nusselt number $(\mathrm{Nu})$ given as

$$
c_{f}=\left.\frac{d w}{d \eta}\right|_{\eta=0,1}, \quad N u=\left.\frac{d \theta}{d \eta}\right|_{\eta=0,1} .
$$

The current density and the total current generated within the MHD flow system are given as

$$
\begin{gathered}
J_{z}=\theta^{\lambda}(L a+w) \quad \text { (Current density), } \\
I_{T}=\int_{0}^{1} \theta^{\lambda}(L a+w) d \eta \quad \text { (Total current). }
\end{gathered}
$$

It is important to note that for a short circuit configuration (where there is no electric field loading) $L a=0$.

\section{Entropy generation analysis}

The convection process in a channel is inherently irreversible and thus causes continuously entropy generation.
The local volumetric rate of entropy generation for a viscous incompressible conducting fluid in the presence of magnetic field and induce electric field is given as ( [22].)

$$
E_{G}=\frac{k}{T_{0}^{2}}\left(\frac{d T}{d y}\right)^{2}+\frac{\mu}{T_{0}}\left(\frac{d u}{d y}\right)^{2}+\frac{\bar{\sigma}\left(E_{z}+u B_{0}\right)^{2}}{T_{0}} .
$$

The first term in equation (10) describes the heat transfer irreversibility while the second and third terms represent irreversibility due to fluid friction and magnetic field respectively. Using equation (6), the dimensionless form of local entropy generation rate in equation (10) is given as

$$
\begin{aligned}
N_{s}= & \frac{T_{0}^{2} a^{2} E_{G}}{k\left(T_{w}-T_{0}\right)^{2}}=\left(\frac{d \theta}{d \eta}\right)^{2} \\
& +\frac{B r}{\Omega}\left[\left(\frac{d w}{d \eta}\right)^{2}+M(L a+w)^{2} \theta^{\lambda}\right],
\end{aligned}
$$

where $\Omega=\left(T_{w}-T_{0}\right) / T_{0}$ is the temperature difference parameter and $\mathrm{Br}=\mathrm{EcPr}$ is the Brinkmann number.

$$
\begin{gathered}
N_{1}=\left(\frac{d \theta}{d \eta}\right)^{2} \quad \text { (Heat transfer Irreversibility) } \\
N_{2}=\frac{B r}{\Omega}\left[\left(\frac{d w}{d \eta}\right)^{2}+H a(L e+w)^{2} \theta^{\lambda}\right]
\end{gathered}
$$

(Fluid friction and magnetic field irreversibility).

The Bejan number Be is define as

$$
B e=\frac{N_{1}}{N_{S}}=\frac{1}{1+\Phi},
$$

where $N_{s}=N_{1}+N_{2}, \Phi=\frac{N_{2}}{N_{1}} \quad$ (Irreversibility Ratio).

The Bejan number $(\mathrm{Be})$ as shown in equation (14) has range $0 \leq B e \leq 1$. Irreversibility is dominated by the combined effects of fluid friction and magnetic fields, if $B e=0$. Irreversibility due to heat transfer dominates the flow system by the virtue of finite temperature differences if $B e=1$.

\section{Results and discussion}

We performed numerical solutions of the above models by chosen some physically meaningful values for the parameters. The results are illustrated graphically in Figures $(3-34)$.

\subsection{Effects of parameter variation on velocity profile}

The key parameters effect on the velocity profiles are presented in Figures $(3-8)$. Generally, we noticed mostly 
negative velocity values at the upper plate. This indicates flow reversal due to a velocity slip. This result agreed with the earlier observation of [3]. It is noticed in Figure 2 that the fluid velocity decreased at both permeable walls and was skewed toward the upper permeable wall due to suction as the as Reynolds number $(R e)$ increases. The electric field loading parameter $(L a)$ effect is observed in Figure 3. Increases in $(L a)$ decrease the fluid velocity in the channel and increase the fluid velocity at the upper permeable wall. Clearly, the velocity decreases with increasing Magnetic field parameter $(M)$ as shown in Figure 4. This tends to slow down the movement of the fluid in channel because of the application of magnetic fields that creates a resistive force that acts in different direction to the fluid motion and thereby cause decrease in the fluid velocity. This observation agreed perfectly with the earlier theoretical and experimental results of $[4-6,11]$ and thus validates our present studies. Figure 5 shows the influence of Navier slip parameter $\gamma_{1}$ at the plates, on the velocity profile. The fluid velocity decreases at both permeable plates as $\gamma_{1}$ increases while Figure 6 shows an increase in fluid velocity at the lower permeable wall with little or no effect at the upper permeable plate as Navier slip parameter $\gamma_{0}$ increases. Effect of the pressure gradient $(G)$ on the velocity profile is shown in Figure 7. Increases in $G$ caused the fluid velocity at the lower plate and in the channel to increase while the fluid velocity at the upper permeable plate decreased due to the reversal flow that occurred. Figure 8 shows the effect of the electrical conductivity variation in$\operatorname{dex}(\lambda)$ on the velocity profile. As $(\lambda)$ increases, the fluid velocity at centerline of the channel increases but no effect is noticed at both plates.

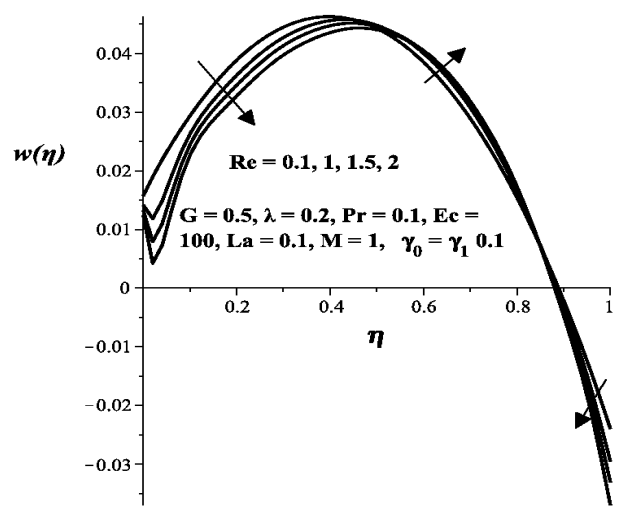

Figure 2: Effect of increasing $R e$ on velocity profile

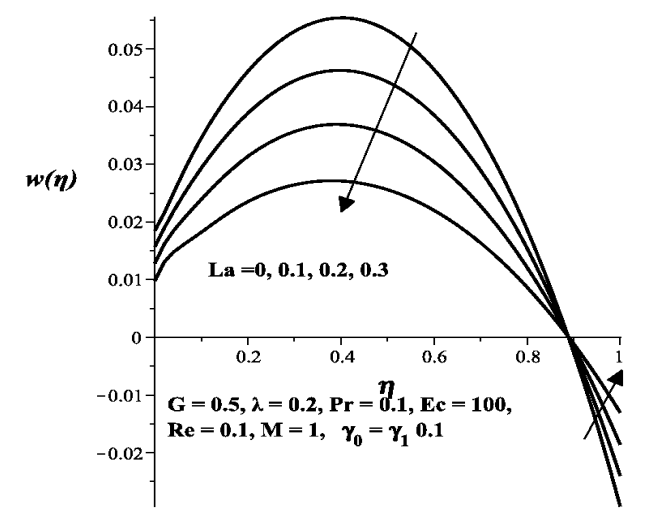

Figure 3: Effect of increasing $L a$ on velocity profile

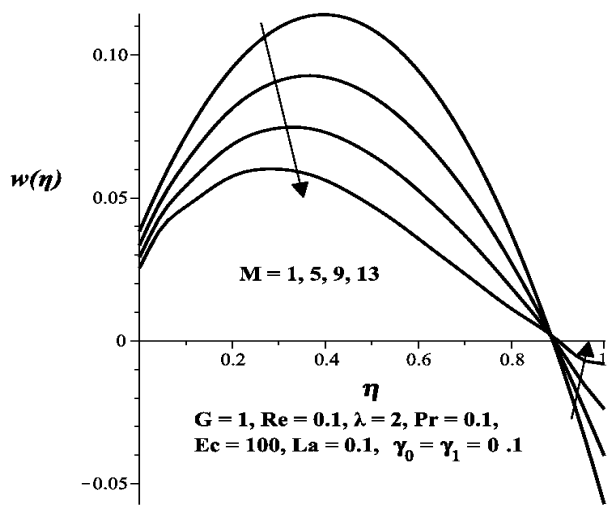

Figure 4: Effect of increasing $M$ on velocity profile

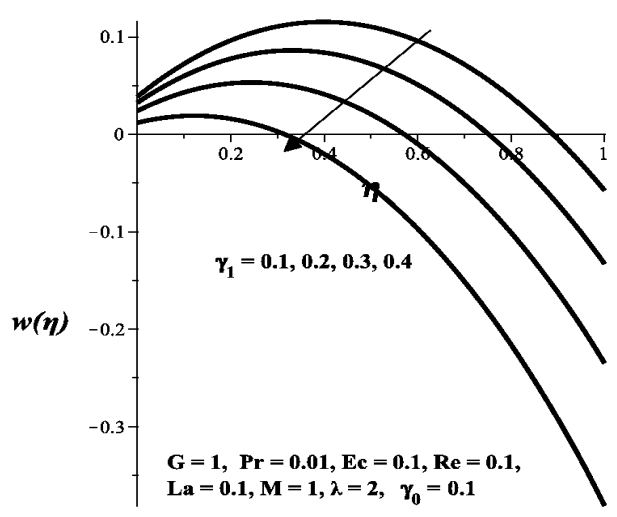

Figure 5: Effect of increasing $\gamma_{1}$ on velocity profile 


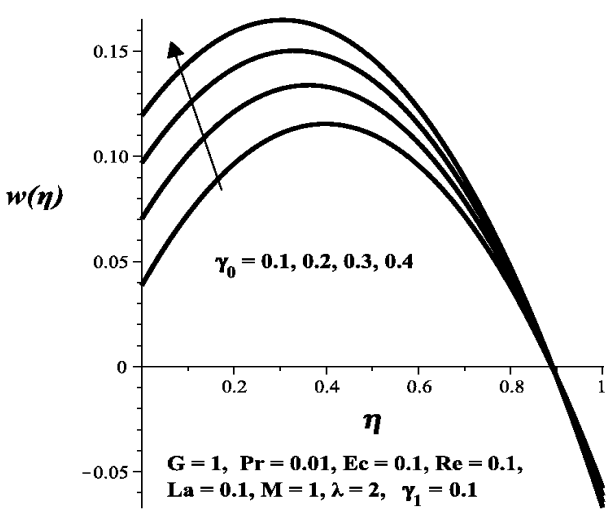

Figure 6: Effect of increasing $\gamma_{0}$ on velocity profile

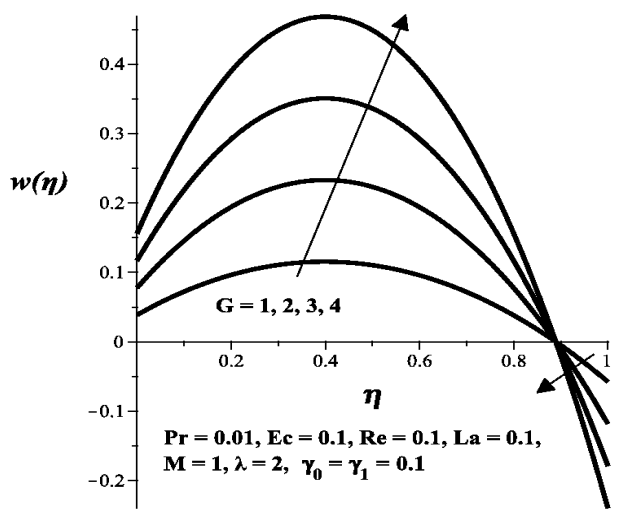

Figure 7: Effect of increasing $G$ on velocity profile

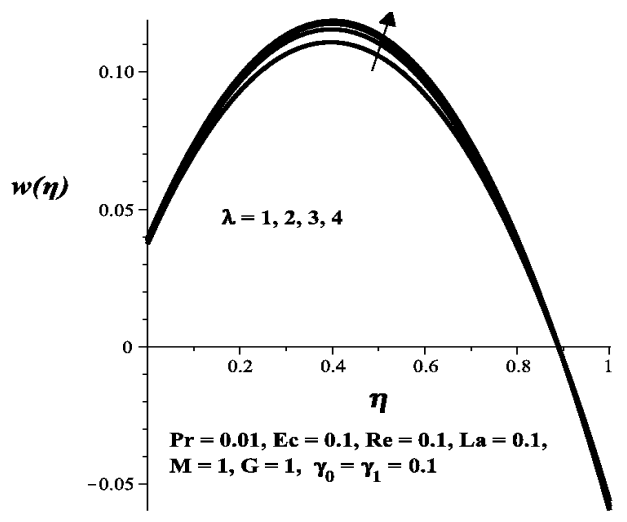

Figure 8: Effect of increasing $\lambda$ on velocity profile

\subsection{Effects of parameter variation on temperature profile}

The influence of various key parameters on the dimensionless temperature distribution are presented in Figures $(9-12)$. Here we see that for varied key parameters the minimum temperature $\theta=0$ at the lower plate and maximum temperature $\theta=1$ at the upper plate. Figure 9 shows the effect of $R e$ on the temperature profile. As $R e$ is increasing, it is noticed that the temperature of the flow decreases at all point. The influence of $\operatorname{Pr}$ on the temperature profile is shown in Figure 10. As $P r$ increases, the fluid element of the molecular motion is lowered and thus the flow field suffers a decrease in temperature at all points. Figures $(11-12)$ show an increase in temperature as the Eckert number $(E c)$ and $L a$ increases at all points.

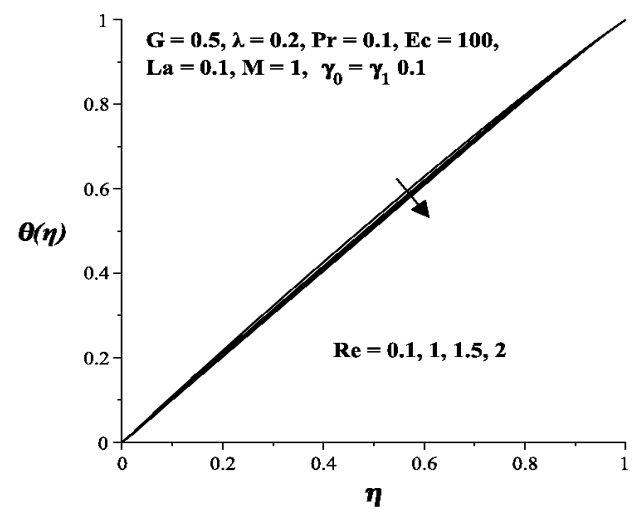

Figure 9: Effect of increasing $R e$ on Temperature profile

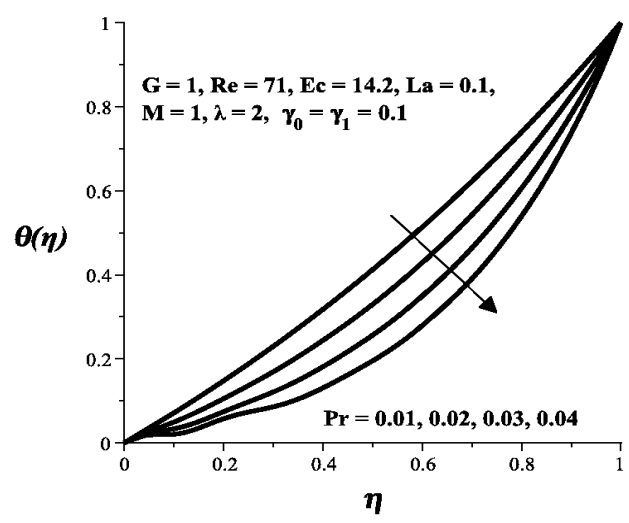

Figure 10: Effect of increasing $\operatorname{Pr}$ on Temperature profile 


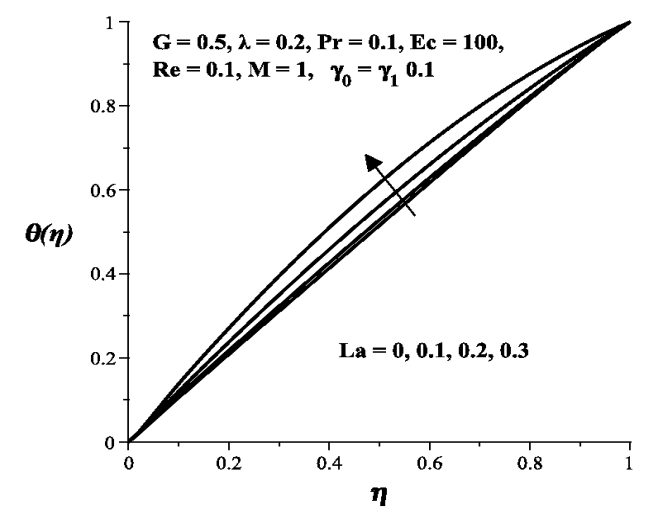

Figure 11: Effect of increasing $L a$ on Temperature profile

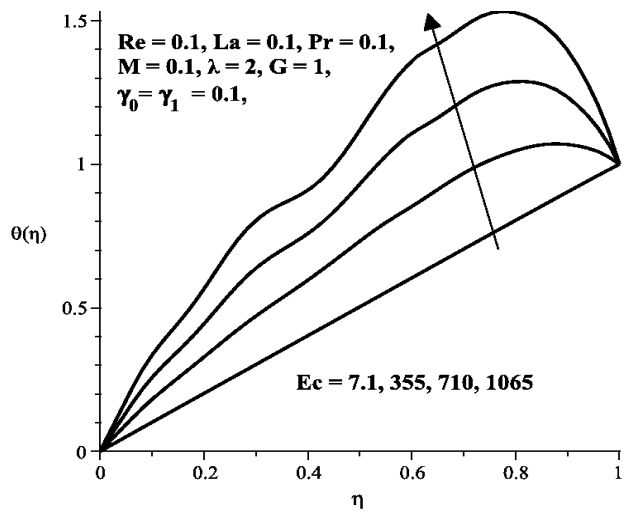

Figure 12: Effect of increasing $E c$ on Temperature profile

\subsection{Effects of parameter variations on current density}

The thermophysical parameters effects on the current density $\left(J_{z}\right)$ are depicted in Figures $(13-19)$. Generally, the influences of these key parameters on current density are asymmetric. Figure 13 illustrates that the current density has a rapidly decreasing nature as $\lambda$ increases in the channel. Figure 14 shows a rapid increase in the current density at the upper plate with increase in $L a$. There is agreement in this observation with the earlier result reported by [20]. Meanwhile, $L e=0$ represents the case of short circuit in the flow field without electric field. Re effects on the current density is presented in Figure 15. As $R e$ increases, a decrease in current density across the channel is noticed. There is no effect on the lower plate but a slight decrease is noticed at the upper plate while $R e=0$ represents the case of impermeable plates. Figure 16 shows that as $\gamma_{0}$ increased, the current density increased in the channel but decreased at the upper plates. The influence of $M$ on the current density is illustrated in Figure 17. It is clear that as $M$ increases, the current density decreases in the channel but increases at the upper plate while an increase in $E c$ (as

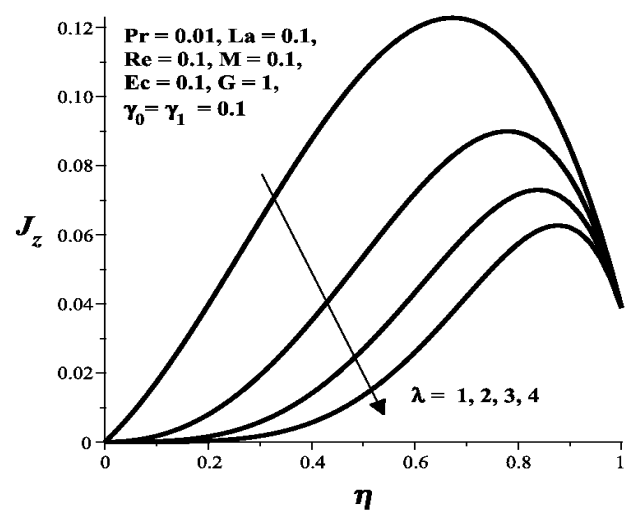

Figure 13: Effect of increasing $\lambda$ on $J_{z}$

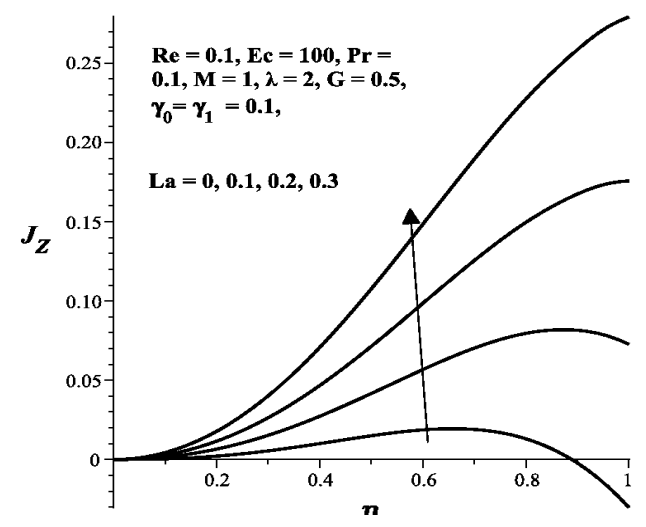

Figure 14: Effect of increasing $L a$ on $J_{z}$

shown in Figure 18), only increases the current density in the channel but has no effect on both the plates. The effect of $\mathrm{Pr}$ is illustrated in Figure 19. It is clear that as $\mathrm{Pr}$ increases, the current density in the channel decreases.

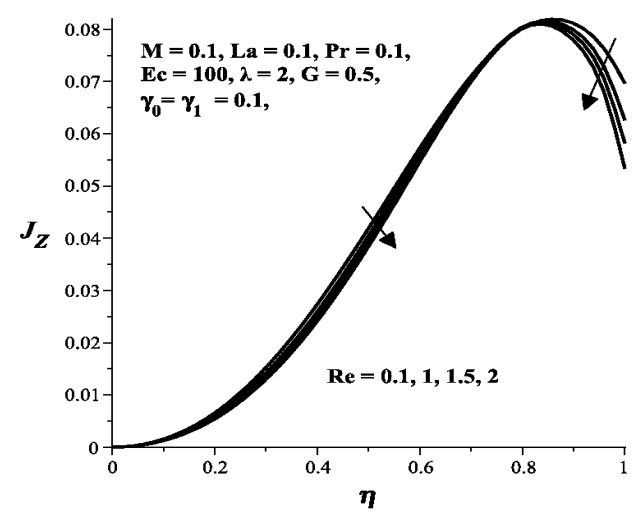

Figure 15: Effect of increasing $\operatorname{Re}$ on $J_{z}$ 


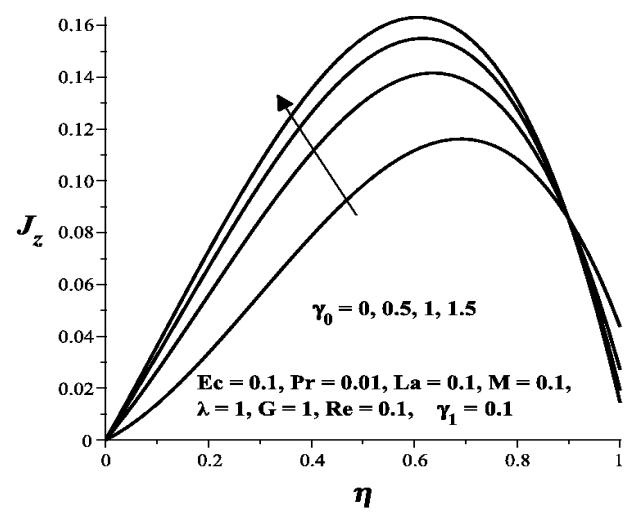

Figure 16: Effect of increasing $\gamma_{0}$ on $J_{z}$

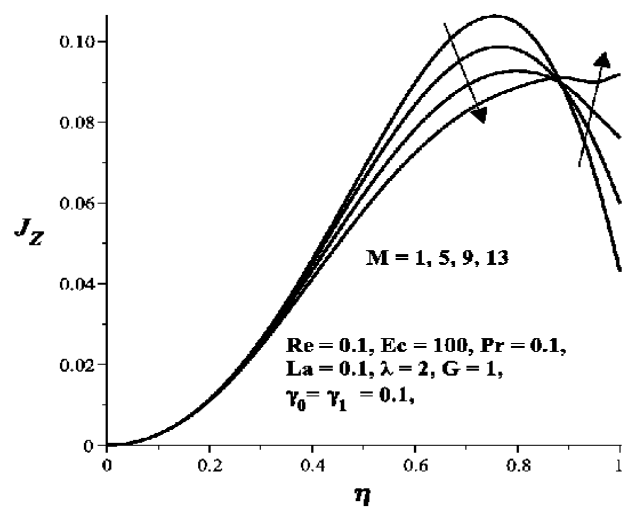

Figure 17: Effect of increasing $M$ on $J_{z}$

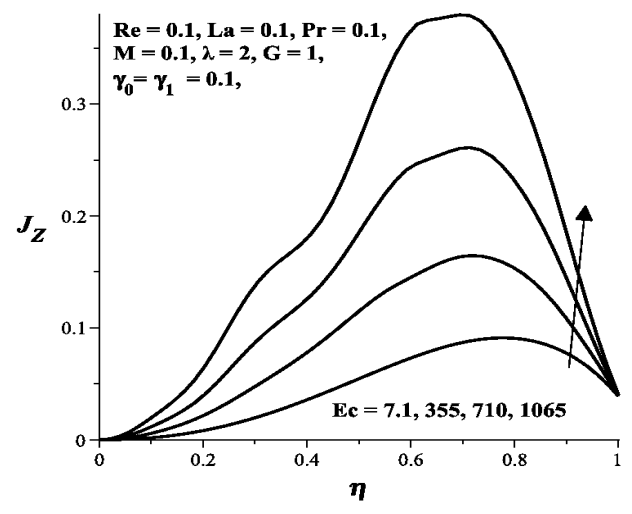

Figure 18: Effect of increasing $E c$ on $J_{z}$

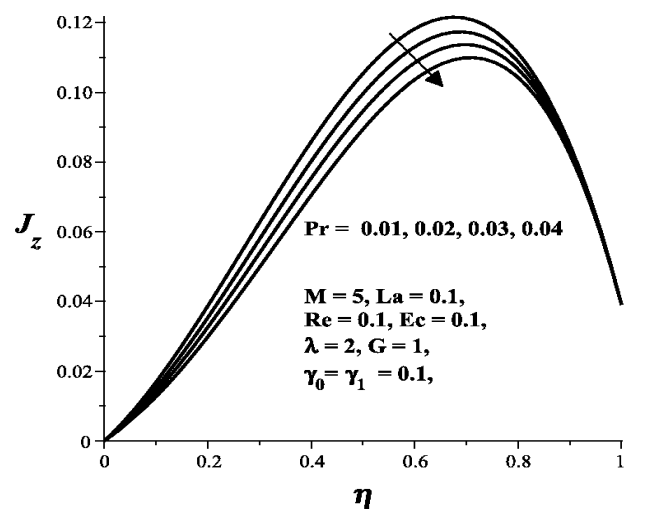

Figure 19: Effect of increasing $\operatorname{Pr}$ on $J_{z}$

\subsection{Effects of parameter variations on entropy generation rate}

Figures $(20-26)$ represent the effect of different values of the key parameters on the entropy generation rate. The effect of $G$ is shown in Figure 20. With an increase in $G$, the entropy generation rate at both upper and lower permeable plates increases. With an increase in $R e$ as shown in Figure 21, it is observed that the entropy generation rate at the lower permeable plate decreases and increases at the upper permeable plate as well. At the centreline of the channel there is no effect. Figure 22 depicts the increase in $\lambda$. As $\lambda$ is increasing, the entropy generation rate at the lower plate up to the point $\eta=0.8$ decreases but as from this point to the upper permeable plate entropy production shoot up and increases. The influence of $L a$ is shown in Figure 23, as $L a$ is increasing, the entropy generation rate in the flow channel increases at all points. Group parameter is an important dimensionless number for entropy generation rate analysis. The relative importance of viscous effects to the temperature gradient entropy generation is determined. Increasing the group parameter $\left(\mathrm{Br} \Omega^{-1}\right.$ ) (as shown in Figure 24),results in the entropy generation rate at both plates increasing. The influence of $M$ on the entropy generation is shown in Figure 25 and as $M$ increases, the entropy generation rate in the channel increases. At both plates, a decrease in the entropy generation rate is noticed. The effect of $\gamma_{0}$ is depicted in Figure 26. As $\gamma_{0}$ increases, it has no effect on the entropy generation at the lower plate but has a decreasing effect at the upper plate. In the channel prior to the upper plate, we noticed an increase in the entropy generation. 


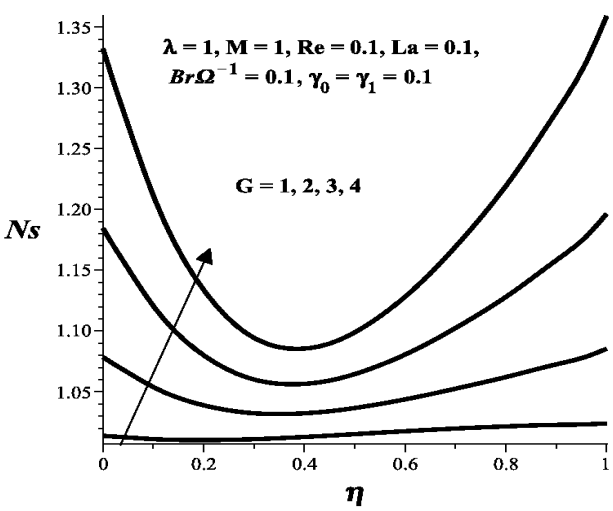

Figure 20: Effect of increasing $G$ on $N s$

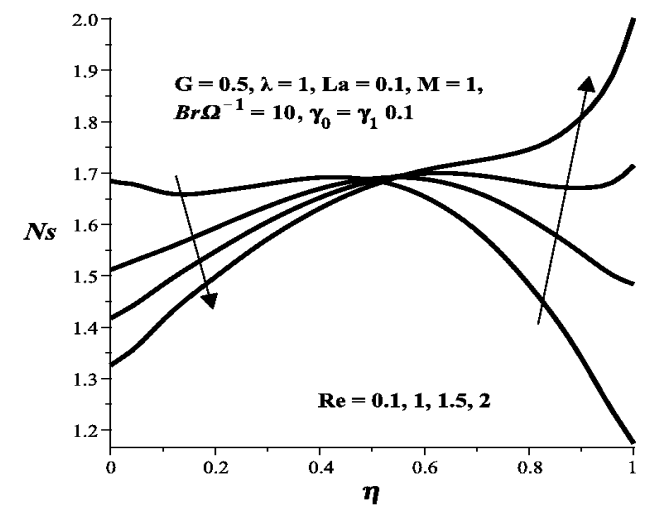

Figure 21: Effect of increasing $\mathrm{Re}$ on $\mathrm{Ns}$

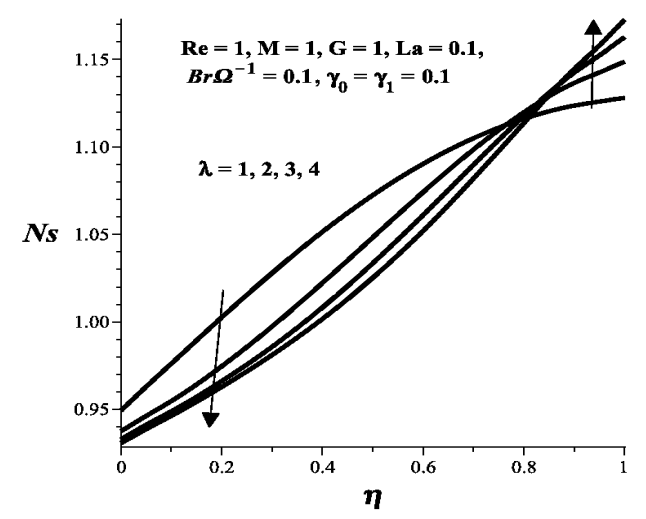

Figure 22: Effect of increasing $\lambda$ on Ns

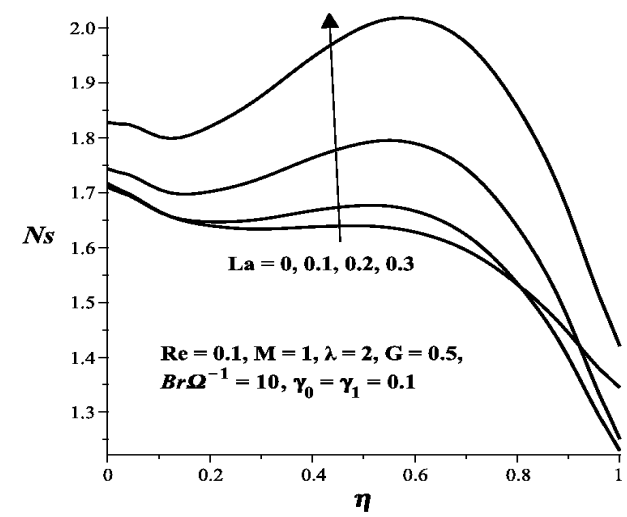

Figure 23: Effect of increasing La on Ns

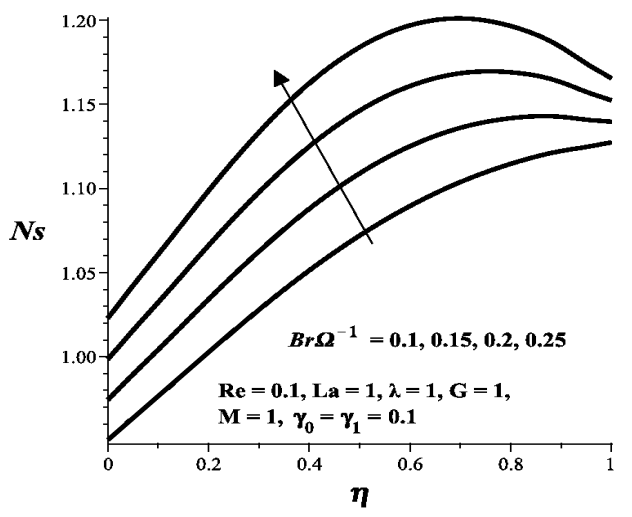

Figure 24: Effect of increasing $\mathrm{Br} \Omega^{-1}$ on $N s$

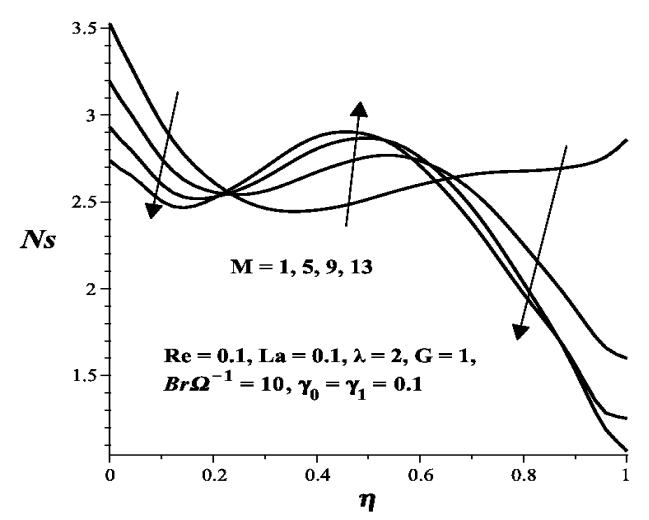

Figure 25: Effect of increasing $M$ on $N s$ 


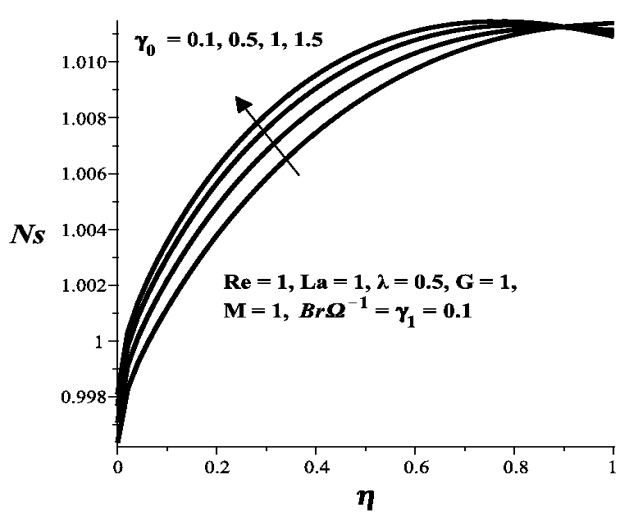

Figure 26: Effect of increasing $\gamma_{0}$ on $\mathrm{Ns}$

\subsection{Effects of parameter variation on Bejan number}

The influence of various parameters on the Bejan number is shown in Figures (27 - 34). With increasing $G$ (as shown in Figure 27), the Bejan number of the flow process decreased. This means that irreversibility due to heat transfer dominates the flow process. The effect of $R e$ is shown in Figure 28. As $R e$ is increasing, the Bejan number at the lower permeable wall is decreasing and the Bejan number at the upper permeable wall is increasing. At point $\eta=0.5$ we noticed no effect on Bejan number. Irreversibility due to fluid friction dominates the lower permeable wall and irreversibility due to heat transfer dominates the upper plate but at the point $\eta=0.5$ both irreversibility due to fluid friction and irreversibility due to heat transfer contributed equally. Figure 29 illustrates the effect of $\lambda$ on the Bejan number. From this Figure, it can be seen that the Bejan number increases with an increase in $\lambda$ in the channel but it decreases with $\lambda$ at the plates. Meanwhile, Figure 30 shows a decrease in the Bejan number with $M$ at upper permeable plate but an increase in the Bejan number at lower permeable plate. The influence of $\mathrm{Br} \Omega^{-1}$ on the Bejan number is illustrated in Figure 31. An increase in $B r \Omega^{-1}$ bolsters the effect of irreversibility due to fluid friction across the flow process leading to a lower Bejan number. The effect of an increase $\gamma_{0}$ on the Bejan number is shown in Figure 32. Initially as $\gamma_{0}$ increases, the Bejan number at the lower plate increased but immediately after $\eta=0.1$, the Bejan number decreases. This implies that irreversibility due to heat transfer dominates the lower permeable plate while irreversibility due to fluid friction dominates the flow after $\eta=0.1$ At $\eta=0.1$ both contributed equally to the flow process. Meanwhile an increase in $\gamma_{1}$, as shown in Figure 33, increased the Bejan number in the entire flow process. This means that irreversibility due to

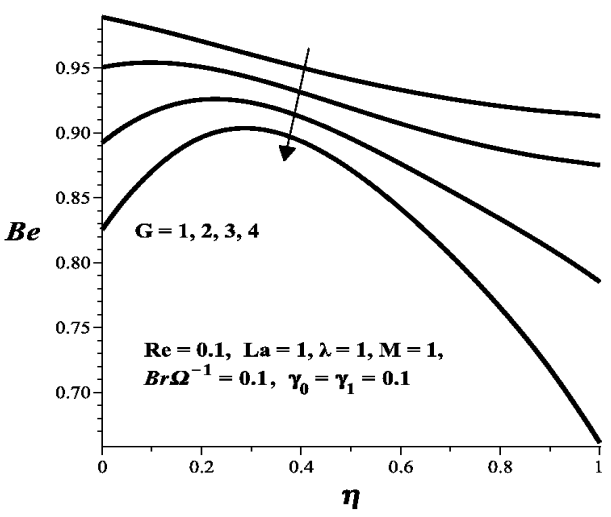

Figure 27: Effect of increasing $G$ on $B e$

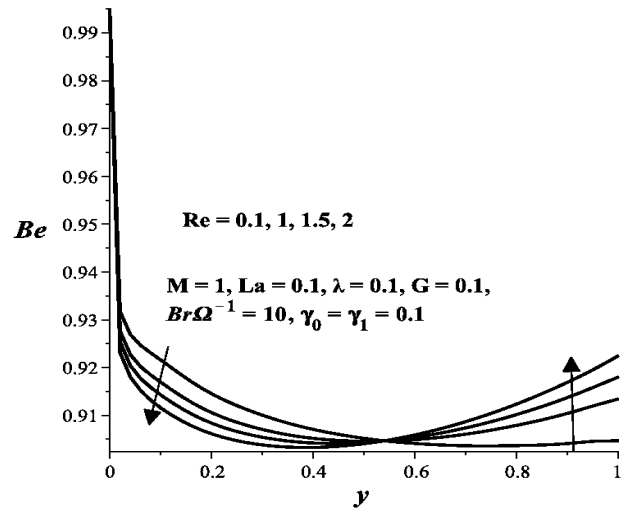

Figure 28: Effect of increasing $R e$ on $B e$

heat transfer dominates the entire flow process. Figure 34 presents the effect of $L a$ on the Bejan number. With increase $L a$, it is noticed that the Bejan number increases at the lower permeable wall and decreases at the upper permeable wall. Within the channel, there also exists cross flow.

\section{Conclusion}

Second law analysis for MHD permeable channel flow with variable electrical conductivity and asymmetric Navier slips is carried out. The velocity and temperature profiles are obtained numerically and used to compute the entropy generation number and current density. The effects of the suction/injection Reynolds number, electric field loading parameter, Magnetic field parameter, Eckert number, Prandtl number and the pressure gradient parameter on velocity, temperature and current density profiles are presented. The influences of these parameters and the dimensionless group parameter on the entropy generation rate and Bejan number are also presented. From the results 


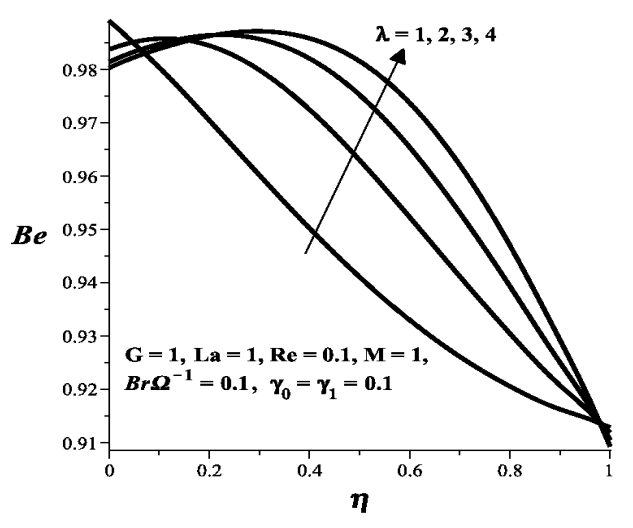

Figure 29: Effect of increasing $\lambda$ on $B e$

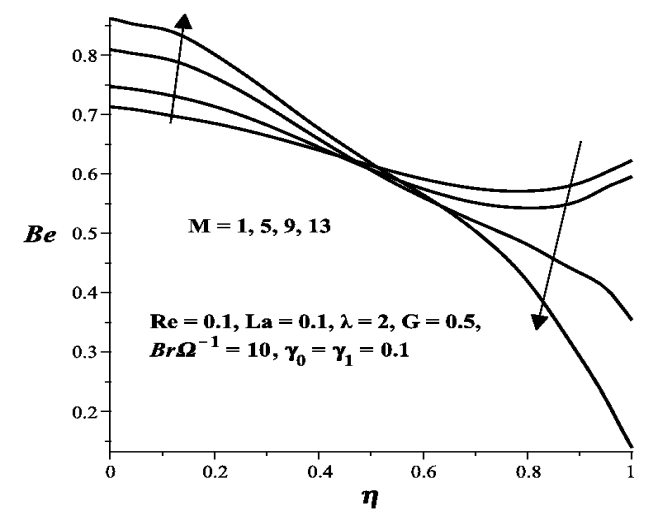

Figure 30: Effect of increasing $M$ on $B e$

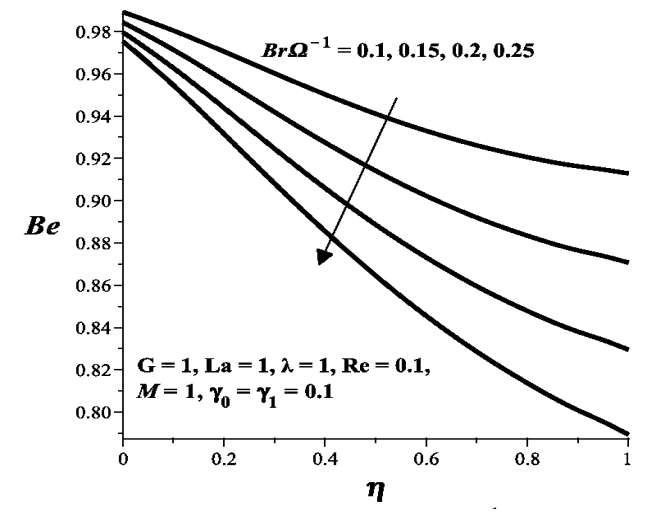

Figure 31: Effect of increasing $\mathrm{Br} \Omega^{-1}$ on $\mathrm{Be}$

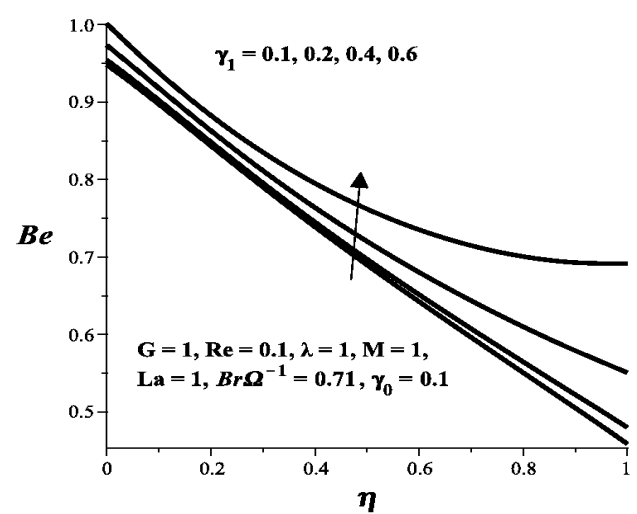

Figure 32: Effect of increasing $R e$ on velocity profile

and subsequent discussions the following conclusions are deduced:

- Increases in $L a, M$, and $\gamma_{1}$ decrease the velocity profile at the centre of the channel while an increase in $\gamma_{0}, G$ and $\lambda$ increases the velocity profile.

- Increases in $R e$ and $P r$ decreases the temperature of the flow process while increases in $L a$ and $E c$ increase the temperature of the flow process.

- Generally, the flow under current density is asymmetric. Increases in $\lambda, R e$ and $P r$ decrease the current density of the flow process while decreases in $L a$ and $E c$, increase the current density. Moreso, increases in $\gamma_{0}$ increase the current density in the channel and decrease the current density at the upper plate. The current density decreases in the channel and increases at the upper plate as $M$ increases.

- Increases in $R e$ and $\lambda$ decrease entropy production rates at the injection plate but increase entropy production at the suction plate. Meanwhile, increases in $G, L a, M, B r \Omega^{-1}$ and $\gamma_{0}$ increase entropy production rates of the flow process.

- Increases in $\gamma_{0}, L a$ and $M$ increase the Bejan number at the injection plate and decrease the Bejan number at suction plate while increases in $R e$ decrease the Bejan number at the injection plate and increase the Bejan number at the suction plate. Increases in $G$ decrease the Bejan number at both plates, similarly for $\lambda$ but the Bejan number increases in the channel. Increases in $\mathrm{Br} \Omega^{-1}$ decrease the Bejan number across the flow while increases in $\gamma_{1}$ increase the Bejan number across the flow. 


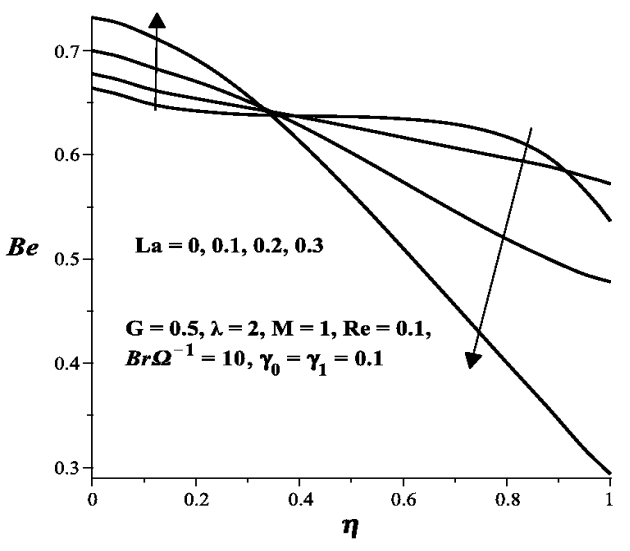

Figure 33: Effect of increasing $L a$ on velocity profile

\section{References}

[1] W.A. Manyonge, D.W. Kiema, C.C.W. Iyaya, Int. J. Pure. Appl. Math., 76, 661 (2012)

[2] O.D. Makinde, P.Y. Mhone, Romania J. Phys., 50, 931 (2005)

[3] O.D. Makinde, E. Osalusi, Romania J. Phys., 51, 319 (2006)

[4] J.D. McWhirter, M.E. Crawford, D.E. Klein, Fusion Sci. Tech. 34, 187 (1998)

[5] S.Y. Smolentsev, A.V. Tananaev, D.A. Dajeh, V.S. Shmarov, Magnetohydrodyn. 33, 155 (1997)
[6] J.C.R. Hunt, R.J. Holroyd, Applications of laboratory and theoretical MHD duct flow studies in fusion reactor technology, UKAEA Culhan Technical Report CLM-R169 (Culham Laboratory, Culham, 1977)

[7] A. Bejan, Adv. Heat Transfer 15, 1 (1982)

[8] A. Bejan, Entropy Generation Minimization (CRC: Boca Raton, FL, USA, 1996)

[9] O.D. Makinde, A.S. Eegunjobi, Entropy 15, 220 (2013)

[10] O. Haddad, A. Mohammad, Mohammad Al-Nimr, Entropy 6, 413 (2004)

[11] S. Mahmud, R.A. Fraser, Energy 28, 1557 (2003)

[12] A.S. Eegunjobi, O.D. Makinde, Math. Probl. Eng. 2013, 630798 (2013)

[13] S. Wijewardane, D.Y. Gosawami, Energy 42, 497 (2012)

[14] Y. Candau, Solar Energy 75, 241 (2003)

[15] A. Aziz, Commun. Nonlinear Sci. Numer. Simul. 15, 573 (2010)

[16] A.S. Eegunjobi, O.D. Makinde, Entropy 33, 692 (2012)

[17] M.J. Martin, I.D. Boyd, J. Thermophys. Heat Transfer 20, 710 (2006)

[18] A.S. Eegunjobi, O.D. Makinde, J. Therm. Sci. Tech. 7, 522 (2012)

[19] Q. He, X. Wang, Z. Angew. Math. Mech. 89, 857 (2009)

[20] O.D. Makinde, O.O. Onyejekwe, J. Magn. Magn. Mater. 323, 2757 (2011)

[21] P.R. Nachtshein, P.Swigert, Satisfaction of the Asymptotic Boundary Conditions in Numerical Solution of the System of Nonlinear Equation of Boundary Layer Type (NASA TND-3004, Washington, USA, 1965)

[22] L.C. Wood, Thermodynamics of Fluid Systems (Oxford University Press, Oxford, 1975) 